\title{
Real-time-guided bone regeneration around standardized critical size calvarial defects using bone marrow-derived mesenchymal stem cells and collagen membrane with and without using tricalcium phosphate: an in vivo micro- computed tomographic and histologic experiment in rats
}

\author{
Khalid Al-Hezaimi ${ }^{1,2}$, Sundar Ramalingam ${ }^{3}$, Mansour Al-Askar ${ }^{2}$, Aws S ArRejaie ${ }^{4}$, Nasser Nooh ${ }^{3}$, Fawad Jawad ${ }^{1}$, \\ Abdullah Aldahmash ${ }^{5}$, Muhammad Atteya ${ }^{5}$ and Cun-Yu Wang ${ }^{6}$
}

The aim of the present real time in vivo micro-computed tomography $(\mu \mathrm{CT})$ and histologic experiment was to assess the efficacy of guided bone regeneration (GBR) around standardized calvarial critical size defects (CSD) using bone marrow-derived mesenchymal stem cells (BMSCs), and collagen membrane (CM) with and without tricalcium phosphate (TCP) graft material. In the calvaria of nine female Sprague-Dawley rats, full-thickness CSD (diameter $4.6 \mathrm{~mm}$ ) were created under general anesthesia. Treatment-wise, rats were divided into three groups. In group 1, CSD was covered with a resorbable CM; in group 2, BMSCs were filled in CSD and covered with CM; and in group 3, TCP soaked in BMSCs was placed in CSD and covered with CM. All defects were closed using resorbable sutures. Bone volume and bone mineral density of newly formed bone (NFB) and remaining TCP particles and rate of new bone formation was determined at baseline, 2, 4, 6, and 10 weeks using in vivo $\mu \mathrm{CT}$. At the 10th week, the rats were killed and calvarial segments were assessed histologically. The results showed that the hardness of NFB was similar to that of the native bone in groups 1 and 2 as compared to the NFB in group 3. Likewise, values for the modulus of elasticity were also significantly higher in group 3 compared to groups 1 and 2 . This suggests that TCP when used in combination with BMSCs and without CM was unable to form bone of significant strength that could possibly provide mechanical "lock" between the natural bone and NFB. The use of BMSCs as adjuncts to conventional GBR initiated new bone formation as early as 2 weeks of treatment compared to when GBR is attempted without adjunct BMSC therapy.

International Journal of Oral Science (2016) 8, 7-15; doi:10.1038/ijos.2015.34; published online 18 December 2015

Keywords: bone marrow-derived mesenchymal stem; collagen membrane; critical size defect; guided bone regeneration; tricalcium phosphate

\section{INTRODUCTION}

There is abundant evidence confirming that guided bone regeneration (GBR) using particulate graft material and resorbable collagen membrane $(\mathrm{CM})$ is a reliable technique to treat and fill osseous defects. ${ }^{1-6}$ Although autogenous bone is considered to be the "gold standard" augmentation material for $\mathrm{GBR}^{5}$; synthetic graft materials such as tricalcium phosphate (TCP) have also been reported to be bioactive and exhibit osteogenic potential. ${ }^{7-9}$ Moreover, the rough surface of CMs augments the overall effect of GBR by providing a surface for osteoblastic cell attachment, supporting osteoid formation and stabilizing the graft material. ${ }^{10}$
The bone marrow contains hematopoietic stem cells and approximately $0.01 \%$ stromal cells named bone marrow-derived mesenchymal stem cells (BMSCs). When expanded in vitro under appropriate conditions, BMSCs have been reported to differentiate into osteoblasts, adipocytes, and/or chondroblasts. ${ }^{11}$ In a clinical study, Gimbel et al. ${ }^{12}$ showed that the addition of bone marrow aspirates soaked in absorbable collagen sponges to patients with alveolar clefts, yielded bone regeneration similar to iliac bone grafts, and reduced postoperative discomfort. Furthermore, two-dimensional (2D) histologic studies have shown that GBR using BMSCs promote neovascularization and bone formation and is also useful in vertical ridge augmentation. ${ }^{13-14}$

${ }^{1}$ Engineer A.B. Growth Factors and Bone Regeneration Research Chair and 3D Imaging Center, College of Dentistry, King Saud University, Riyadh, Saudi Arabia; ${ }^{2}$ Department of Periodontics and Community Dentistry, College of Dentistry, King Saud University, Riyadh, Saudi Arabia; ${ }^{3}$ Department of Oral and Maxillofacial Surgery, College of Dentistry, King Saud University, Riyadh, Saudi Arabia; ${ }^{4}$ Department of Substitutive Dental Sciences, College of Dentistry, University of Dammam, Dammam, Saudi Arabia; ${ }^{5}$ Stem Cell Unit, Department of Anatomy, College of Medicine, King Saud University, Riyadh, Saudi Arabia and ${ }^{6}$ Division of Oral Biology and Medicine, School of Dentistry, University of California at Los Angeles, Los Angeles, USA

Correspondence: Dr K Al-Hezaimi, Engineer A.B. Growth Factors and Bone Regeneration Research Chair and 3D Imaging Center, Department of Periodontics and Community Dentistry, College of Dentistry, King Saud University, Riyadh 11545, Saudi Arabia

E-mail: hezaimik16@gmail.com

Accepted 3 June 2014 
Although 2D histologic analysis has been traditionally used to assess the outcome of $\mathrm{GBR}^{14-17}$; three-dimensional (3D) micro-computed tomography $(\mu \mathrm{CT})$ is an advancement in modern medical imaging technology. Studies ${ }^{18-21}$ have employed ex vivo $\mu \mathrm{CT}$ imaging to assess alveolar bone remodeling and GBR in animal models. For example, in an exvivo $\mu \mathrm{CT}$ experiment on beagle dogs, Al-Hazmi et al. ${ }^{19}$ assessed the outcome of GBR (with and without adjunct growth factor (GF) therapy) around experimental periodontal bony defects. The ex vivo $\mu \mathrm{CT}$ results showed significantly more newly formed bone (NFB) in defects treated using GBR with adjunct GF therapy compared to defects treated using traditional GBR techniques. ${ }^{19}$ Although the results of this 16-week experiment are laudable; ${ }^{19}$ the real time in which bone graft particles began to mineralize and earliest evidence of NFB remained unknown.

In vivo $\mu \mathrm{CT}$ is a step further in modern $3 \mathrm{D}$ imaging technology that allows tissue imaging and assessment in real time. Although histologic results have shown the appearance of NFB within 4-8 weeks of GBR; it is tempting to hypothesize that in vivo $\mu \mathrm{CT}$ imaging shows the precise duration in real time in which NFB fills osseous defects. The aim of the present study was to assess GBR around standardized critical size calvaria defects (CSD) using BMSCs and CM with and without TCP granules using $3 \mathrm{D}$ real time in $\mu \mathrm{CT}$ and traditional $2 \mathrm{D}$ histology.

\section{MATERIAL AND METHODS}

\section{Ethical guidelines}

Prior to initiation of the research study, ethical approval was obtained from the research ethics review committee of the Engineer Abdullah Bugshan Research Chair for Growth Factors and Bone Regeneration (GFBR), College of Applied Medical Science, King Saud University, Riyadh, Saudi Arabia.

\section{Study animals}

Nine female Sprague-Dawley rats with age and weight ranging between 6 weeks and $300 \mathrm{~g}$, respectively were used. All subjects were housed in the animal facility of the Engineer Abdullah Bugshan Research Chair for GFBR, 3D Imaging, and Biomechanical Laboratory, College of Applied Medical Sciences, King Saud University, Riyadh, Saudi Arabia, for at least 7 days preoperatively. All subjects were kept in individual cages at a constant temperature of $22{ }^{\circ} \mathrm{C}$ and maintained with a light cycle of $12 \mathrm{~h}$ (6:00-18:00) with access to drinking water and to a standard laboratory diet ad libitum.

\section{Harvesting and culturing rat BMSCs}

Rat BMSCs were harvested from 10-week-old female Sprague-Dawley rats. Under general anesthesia (GA) and aseptic conditions, the tibia and femur were dissected from the hind limbs bilaterally. After dissection of the bones, the rats were euthanized by anesthetic over dosage. Immediately after dissection, the tibia and femur were transferred into sterile containers with phosphate-buffered saline (PBS) and the container was wrapped with ice during transportation to the stem cell laboratory, King Saud University, Riyadh, Saudi Arabia. The whole bone marrow plugs were flushed from the dissected tibia and femur, by means of a 28 -gauge needle and $1 \mathrm{~mL}$ syringe loaded with culture medium. A needle was inserted into the bone and cells were aspirated followed by several flushes through the bone, until all the bone marrow was flushed out of the bone. A similar procedure was performed from the other end of the bone as close to the tip as possible. Bone marrow cells were centrifuged; pellets were resuspended in red blood cell lysis buffer (Sigma-Aldrich Chemie GmbH, Taufkirchen, Germany) and incubated at room temperature for $5 \mathrm{~min}$. After being washed with PBS, the pellets were resuspended in culture medium. The cell count was performed manually using a microscope in a hemocytometer (Neubauer chamber, Thomas Scientific, Swedesboro, NJ, USA) and furthermore the viability was assessed by Trypan Blue dye exclusion test. The cells were plated in $25 \mathrm{~cm}^{2}$ tissue culture flasks made of polystyrene plastic (Nunclon; Sigma-Aldrich Chemie $\mathrm{GmbH}$, Taufkirchen, Germany) at a density of $10^{5}$ cells per $\mathrm{cm}^{2}$. All non-adherent cells were removed after $48 \mathrm{~h}$. Cells were fed with new culture medium for every 3-4 days until they reached $80 \%-90 \%$ confluence.

The cells were cultured in Dulbecco's modified Eagle's medium (DMEM; supplemented with D-glucose $4500 \mathrm{mg} \cdot \mathrm{L}^{-1}, 4 \mathrm{mmol} \cdot \mathrm{L}^{-1}$ $\mathrm{L}$-glutamine, and $110 \mathrm{mg} \cdot \mathrm{L}^{-1}$ sodium pyruvate), $10 \%$ fetal bovine serum, $1 \times$ penicillin-streptomycin, and non-essential amino acids (all purchased from Gibco-Invitrogen, Grand Island, NY, USA). The culture was maintained at $37{ }^{\circ} \mathrm{C}$ in a humidified $5 \% \mathrm{CO}_{2}$ incubator. The cells were plastic adherent with a fibroblast-like spindle-shaped morphology. When the cells reached $80 \%-90 \%$ confluence, the cells were trypsinized with $0.25 \% \operatorname{trypsin} / 1 \mathrm{mmol} \cdot \mathrm{L}^{-1}$ ethylenediaminetetraacetic acid (EDTA) (Invitrogen, Darmstadt, Germany). Stem cells at a concentration of $1 \times 10^{6}$ cells were prepared for the defect treatment. This was achieved by obtaining a dilution of $6 \times 10^{6}$ BMSCs per $\mathrm{mL}$ of PBS. Approximately $0.15-0.2 \mathrm{~mL}$ of the prepared suspension was used per defect by using a $1 \mathrm{~mL}$ syringe.

\section{Surgical protocol}

All surgical protocols were carried out under GA which was administered using an intraperitoneal injection of a combination of ketamine chloride $\left(70 \mathrm{mg} \cdot \mathrm{kg}^{-1}\right)$ and xylazine chloride $\left(6-9 \mathrm{mg} \cdot \mathrm{kg}^{-1}\right)$. Postanesthesia, dorsal skin covering the scalp was shaved and disinfected with $10 \%$ povidone-iodine solution. A 15 -mm-long midline cutaneous straight incision was released along the sagital suture over the parietal bone of the scalp following which the skin and underlining tissues including the musculature and the periosteum were reflected bilaterally to expose the calvaria (Figure 1a and 1b). A trephine drill (ACE Surgical Supply, Brockton, MA, USA) with an outer diameter of $4.6 \mathrm{~mm}$, was used (with saline irrigation) to create a full-thickness CSD defect on the parietal region lateral to the sagital suture (Figure 1b). Full-thickness bone (including the outer and inner cortices) was carefully removed to prevent damage to the dura (Figure 1c) and a resorbable CM (Bio-Gide, Geistlich Biomaterials, Wolhusen, Switzerland) was placed over the defect (Figure 1d). The flaps were sutured using resorbable sutures (VICRYL (Polyglactin 910) Suture; Ethicon, Somerville, NJ, USA) (Figure 1e).

\section{Animal grouping}

The animals were randomly divided into three groups (three rats per group) by picking a paper from an opaque bag marked either "group 1", "group 2," or "group 3". Group 1 (control): CSD was covered with CM (Bio-Gide, Geistlich Biomaterials, Wolhusen, Switzerland); group 2: CSD was filled with BMSC and covered with CM and group 3: CSD was filled with TCP granules (Ceros TCP granules; Mathys, Bettlach, Switzerland) soaked in BMSC and the defect was covered with CM (BioGide, Geistlich Biomaterials, Wolhusen, Switzerland).

\section{Postoperative management}

A single intramuscular injection of $2.5 \mathrm{mg} \cdot \mathrm{kg}^{-1}$ amoxicillin (Betamox LA; Norbrook Laboratories, Newry, Northern Ireland) and $50 \mathrm{mg} \cdot \mathrm{mL}^{-1}$ solution of analgesic (Metamizol; Vemedim, Can Tho City, Vietnam) was administered to each subject immediately after surgery. All animals were kept under observation until recovery from GA following which they were returned to their individual cages. 

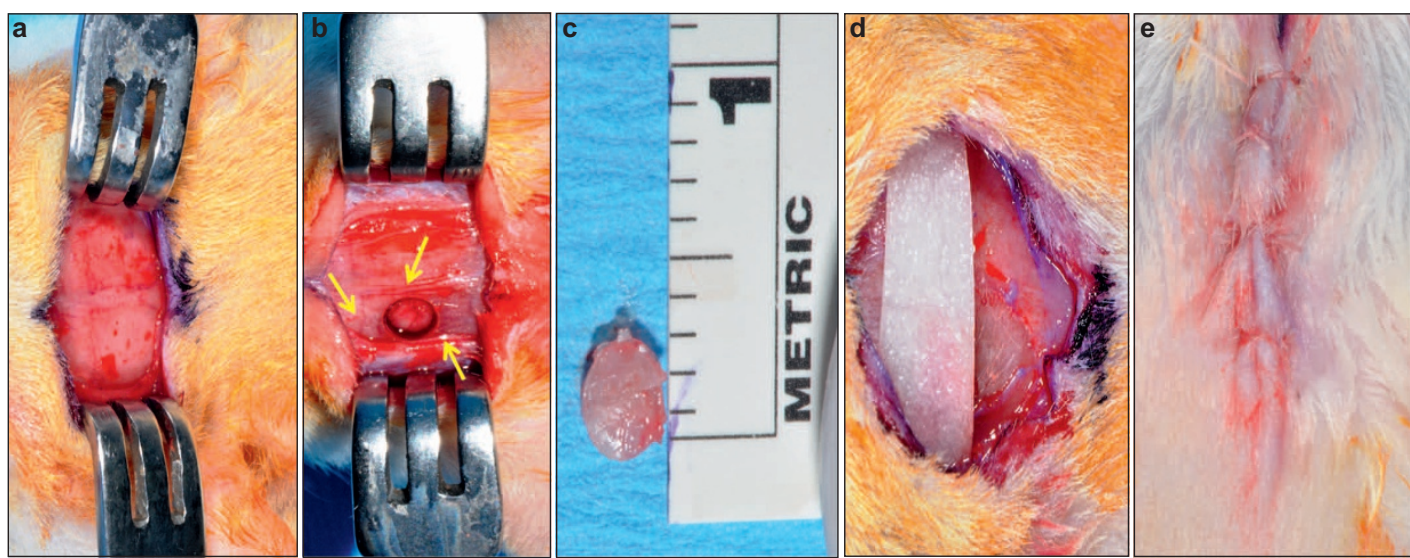

Figure 1 Surgical protocol. (a) Reflection of a 15-mm-long midline cutaneous straight incision along the sagital suture over the parietal bone of the scalp and the skin and underlining tissues including the musculature and the periosteum were reflected bilaterally to expose the calvarium. (b) Using a trephine drill, a full-thickness CSD was created on the parietal region lateral to the sagital suture. (c) Full-thickness bone (including the outer and inner cortices) was carefully removed to prevent damage to the dura. (d) A resorbable CM was placed over the defect. (e) Flaps were sutured using resorbable sutures. CM, collagen membrane; CSD, critical size calvarial defect.

\section{Real-time in vivo microcomputed tomography}

$\mu \mathrm{CT}$ analysis was performed by one investigator who was blinded to the study groups to which the animals belonged. Under GA (as described above), cranial bones were assessed three dimensionally using in vivo $\mu \mathrm{CT}$ (SkyScan 1176; Bruker Micro-CT, Kontich, Belgium). Subjects were positioned in the in vivo $\mu \mathrm{CT}$ scanner in a craniocaudal orientation with suitable stabilization of the head and the body. The subjects were placed in the polystyrene support of the in vivo $\mu \mathrm{CT}$ scanner (SkyScan 1176; Bruker Micro-CT, Brussels, Belgium) and scanned at $65 \mathrm{kV} / 385 \mathrm{~mA}$ source voltage/current, with a 1-mm-aluminum filter. The pixel size (resolution), rotation step, and exposure time were $35 \mu \mathrm{m}, 0.6^{\circ}$ over $360^{\circ}$, and $400 \mathrm{~ms}$, correspondingly. Datasets were reconstructed using a software program (NRecon software, SkyScan; Bruker Micro-CT, Kontich, Belgium). Moderate beam hardening was applied in the reconstruction process. The $\mu \mathrm{CT}$ analysis was performed with a software program (CTAN, SkyScan; Bruker Micro-CT, Kontich, Belgium). The Hounsfield unit (HU) and bone mineral density (BMD) calibrations were first applied to the dataset. Two phantoms of calcium density 0.25 and $0.75 \mathrm{~g} \cdot \mathrm{mm}^{-3}$ and $2 / 4 \mathrm{~mm}$ in diameter (Gloor Instruments AG, Kloten, Switzerland) were scanned in a $10 \mathrm{~mL}$ Falcon tube filled with purified water using the scanning parameters described previously. Manufacturer's HU and BMD calibration procedures were then followed. The region of interest was chosen as a sleeve around NFB and bone particles individually with $0.5 \mathrm{~mm}$ of thickness. Afterwards, the bone volume (BV), percent bone volume (BV/TV), and BMD of bone tissue were measured for each dataset and the remaining particles percentages were taken by subtracting the percent bone volume (BV/TV) of the bigger amount of bone particles from the percent bone volume (BV/TV) of total amount of bone particles. Volumes of NFB and remaining bone particles were measured in cubic millimeters $\left(\mathrm{mm}^{3}\right)$ and BMD was measured in grams per cubic millimeters $\left(\mathrm{g} \cdot \mathrm{mm}^{-3}\right)$.

$\mu \mathrm{CT}$ imaging was performed at baseline and at $2,4,6$, and 10 weeks post-operatively. 3D images of the defect area were constructed using a computer program (Insta Recon Software; EnterpriseWorks, Champaign, IL, USA). Thirty percent beam hardening effect reduction and $12 \%$ ring artifact correction was used to produce the precise image cross section.

\section{Euthanasia}

After the 10th week in vivo $\mu \mathrm{CT}$ scan, all animals were killed. The rats were placed in a euthanasia chamber for $5 \mathrm{~min}$ that was filled with $100 \%$ diethyl ether.

\section{Histology}

Histologic investigations focused mainly on bone regeneration and its location in relation to the defect and/or the grafted bone particles. In addition, a qualitative assessment of the integration of the NFB to the native bone (NB), the presence of remaining TCP particles, evidence of inflammatory reactions and/or necrosis, connective tissue (CT) bridging the defect, and encapsulating fibrous tissue were performed. The following parameters defined NFB from NB (i) the absence of organized lamellated appearance of mature bone; (ii) increased cellularity and evidence of neovascularization; (iii) random display of osteocytes (as compared to being oriented with their long axes parallel to lamellae in mature bone); and (iv) intense staining of matrix with hematoxylin $(\mathrm{H})$ instead of eosin (E).

Briefly, the entire calvarium, extending from the frontal bone to the occipital bone anterio-posteriorly and between the bilateral temporoparietal sutures medio-laterally (including its respective periosteal and dural linings) was harvested and fixed in 10\% neutral buffered formaldehyde solution. Specimens were then decalcified using EDTA solution $\left(0.5 \mathrm{~mol} \cdot \mathrm{L}^{-1}\right)$ at $\mathrm{pH} 8$ for 4 weeks and embedded in paraffin. From each specimen, $4 \mu \mathrm{m}$ sections were prepared with an orientation paralleling the sagital suture before being mounted on slides. For qualitative analysis, the slides were stained with H\&E. Histologic sections were viewed under a light microscope (OLYMPUS CX41; Olympus, Tokyo, Japan) at $\times 4$, $\times 10$, and $\times 40$ magnifications. Images of histologic sections were obtained using a live view digital single-lens reflex camera (OLYMPUS E330; Olympus, Yokyo, Japan). ${ }^{22-23}$

\section{Biomechanical assessment of bone hardness}

The rat skulls from the three groups were harvested 10 weeks after bone repair and healing. The specimens were subjected to indentation to measure their hardness along with other parameters. The specimen's surface was polished down gradually with $1 / 4 \mu \mathrm{m}$ diamond paste slurry. The sample was then placed in a Hysitron TI 750 Ubi (Hysitron, Minneapolis, MN, USA). Each specimen was mounted on self-polymerizing resin (VariDur 10; Buehler, Lake Bluff, IL, USA) and subjected to a fine special silicon carbide grinding paper polishing (EcoMet 250 Grinder Polisher; Buehler, Lake Bluff, IL, USA), dried, and treated with absolute alcohol to decontaminate the surface. Once mounted and secured, the instrument's optical microscope was used to identify the areas of defect for testing. Once the testing location was identified optically, it was refined 
using in situ scanning probe microscopy (SPM) imaging present along with the diamond Berkovich indentation probe (Hysitron, Minneapolis, MN, USA). The bone surface was tested using load controlled quasi-static nanoindentation to obtain the mechanical properties. A series of partial unloads tests were conducted on each area of interest of the specimens. These partial unload tests consisted of 1000 to $3000 \mu \mathrm{N}$ load force. During indentation process, a record of the depth of penetration was made and the area of the indent was determined using the geometry of the indentation tip. A record of these values was plotted on a graph to create a load displacement curve. This curve was used to extract mechanical properties of the material. Hardness and reduced modulus were calculated at each load from the resulting force versus displacement curves and averaged together. After completion of indents, samples were reimaged using in situ SPM imaging technique to ensure proper placement of the indents and an image was produced.

\section{Statistical analysis}

Statistical analysis was performed using the SPSS software (Version 18; SPSS, Chicago, IL, USA). BV and BMD of NFB and remaining TCP particles among the groups were compared using one-way analysis of variance. For multiple comparisons, Bonferroni post hoc test was used. Level of significance was set at $P<0.05$.

\section{RESULT}

In vivo micro-computed tomography

- Group 1 (control)

BMD-NFB was significantly high at 6 weeks $\left((0.32 \pm 0.002) \mathrm{g} \cdot \mathrm{mm}^{-3}\right)$ $(P<0.01)$ and 10 weeks $\left((0.49 \pm 0.001) \mathrm{g} \cdot \mathrm{mm}^{-3}\right)(P<0.01)$ compared to baseline $\left(0 \mathrm{~g} \cdot \mathrm{mm}^{-3}\right)$. At 10 weeks $\left((0.49 \pm 0.001) \mathrm{g} \cdot \mathrm{mm}^{-3}\right)$, BMD-NFB was significantly high as compared to 2 weeks $\left((0.06 \pm 0.01) \mathrm{g} \cdot \mathrm{mm}^{-3}\right)$. There was no significant difference in BV at all the time intervals (Table 1 and Figure 2a).

Table 1 Mean \pm standard deviation of bone volume and bone mineral density of newly formed bone in the group 1

\begin{tabular}{|c|c|c|}
\hline \multirow[b]{2}{*}{ Timeline } & \multicolumn{2}{|c|}{ Newly formed bone } \\
\hline & Bone volume $/ \mathrm{mm}^{3}$ & Bone mineral density $/\left(\mathrm{g} \cdot \mathrm{mm}^{-3}\right)$ \\
\hline Baseline & 0 & $0 * \dagger$ \\
\hline 2 weeks & $0.36 \pm 0.60$ & $0.06 \pm 0.01^{*}$ \\
\hline 4 weeks & $0.98 \pm 0.60$ & $0.12 \pm 0.11$ \\
\hline 6 weeks & $1.80 \pm 0.26$ & $0.32 \pm 0.002 *$ \\
\hline 10 weeks & $2.90 \pm 0.36$ & $0.49 \pm 0.001^{\dagger *}$ \\
\hline
\end{tabular}

$* P<0.01,{ }^{\dagger} P<0.01,{ }^{\star} P<0.01$
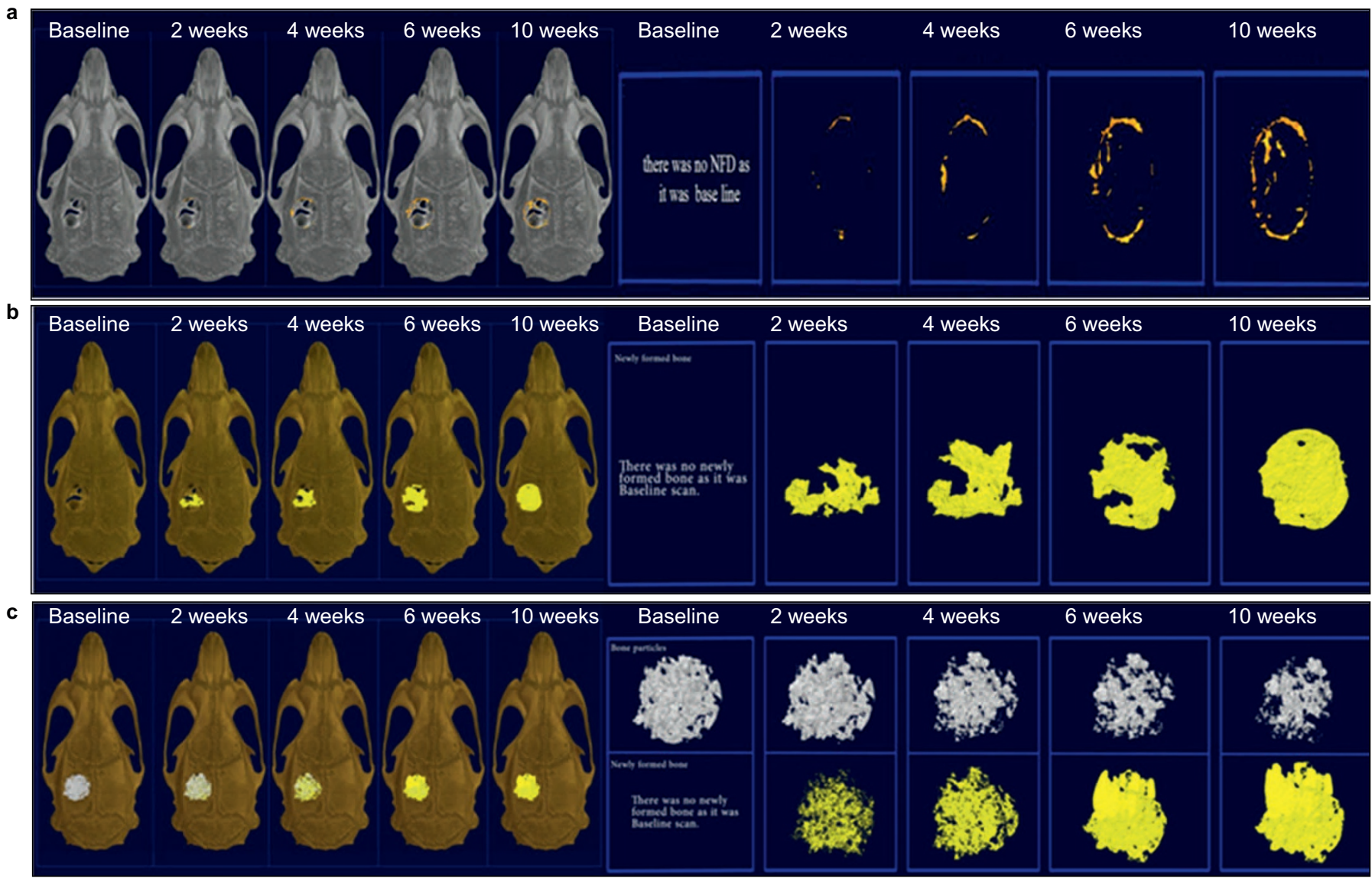

Figure 2 In vivo $\mu \mathrm{CT}$ images of rat calvarial models with critical size defects scanned at 2-, 4-, 6-, and 10-week intervals. (a) Control group shows traces of new bone formation over the study period. (b) Formation of new bone (in yellow) at 2, 4, 6, and 10 weeks in critical size defects filled with MSCs and covered with a resorbable CM. (c) Formation of new bone (in yellow) at 2, 4, 6, and 10 weeks in critical size defects filled with $\beta$-TCP soaked in MSCs and covered with a resorbable CM. CM, collagen membrane; MSC, mesenchymal stem cell; TCP, tricalcium phosphate. 
Table 2 Mean \pm standard deviation of bone volume and bone mineral density of newly formed bone in group 2

\begin{tabular}{|c|c|c|}
\hline \multirow[b]{2}{*}{ Timeline } & \multicolumn{2}{|c|}{ Newly formed bone } \\
\hline & Bone volume $/ \mathrm{mm}^{3}$ & Bone mineral density $/\left(\mathrm{g} \cdot \mathrm{mm}^{-3}\right)$ \\
\hline Baseline & $0 *$ *t\$s & $0^{\dagger+1+s s}$ \\
\hline 2 weeks & $1.16 \pm 0.45 * 1 \%$ & $0.24 \pm 0.05^{11199 \# \#}$ \\
\hline 4 weeks & $2.01 \pm 0.80^{\dagger 1 \#}$ & {$\left[0.31 \pm 0.05^{\dagger+11}\right.$} \\
\hline 6 weeks & $3.90 \pm 0.60$ & *** $0.33 \pm 0.08$ to \\
\hline 10 weeks & *** $7.02 \pm 0.50^{59 \#}$ & L $0.39 \pm 0.05^{\S \S \# \#}$ \\
\hline
\end{tabular}

${ }^{*} P<0.05,{ }^{\dagger} P<0.01,{ }^{\sharp} P<0.01,{ }^{\S} P<0.01,{ }^{~} P<0.01,{ }^{\circledR} P<0.01,{ }^{\#} P<0.01$,

${ }^{* *} P<0.05,{ }^{\dagger \dagger} P<0.05,{ }^{4 \star} P<0.01,{ }^{\S \S} P<0.01,{ }^{\text {"I }} P<0.01,{ }^{\circ "} P<0.01$,

\#\# $P<0.01,{ }^{* * *} P<0.01$.

- Group 2 (BMSCs filled in the critical size defect and covered with a resorbable $\mathrm{CM}$ )

BV-NFB was significantly high at 2 weeks $\left((1.16 \pm 0.45) \mathrm{mm}^{3}\right)$ $(P<0.05), 4$ weeks $\left((2.01 \pm 0.80) \mathrm{mm}^{3}\right)(P<0.01), 6$ weeks $\left((3.90 \pm 0.60) \mathrm{mm}^{3}\right)(P<0.01)$, and 10 weeks $\left((7.02 \pm 0.50) \mathrm{mm}^{3}\right)$ $(P<0.01)$ compared to baseline $\left(0 \mathrm{~mm}^{3}\right)$. At 10 weeks, BV-NFB was significantly high compared to 2 weeks $(P<0.01)$, 4 weeks $(P<$ $0.01)$, and 6 weeks $(P<0.05)$ (Table 2 and Figure $2 b)$.

BMD-NFB was significantly high at 4 weeks $\left((0.31 \pm 0.05) \mathrm{g} \cdot \mathrm{mm}^{-3}\right)$ $(P<0.05), 6$ weeks $\left((0.33 \pm 0.08) \mathrm{g} \cdot \mathrm{mm}^{-3}\right)(P<0.01)$, and 10 weeks $\left((0.39 \pm 0.05) \mathrm{g} \cdot \mathrm{mm}^{-3}\right)(P<0.01)$ compared to baseline $\left(0 \mathrm{~g} \cdot \mathrm{mm}^{-3}\right)$. BMD-NFB at 6 weeks was significantly higher than that at 2 weeks $(P<0.01)$. At 10 weeks, BMD-NFB was significantly high compared to 2 weeks $(P<0.01)$ and 4 weeks $(P<0.01)$ (Table 2 and Figure $2 \mathrm{~b})$.

- Group 3 (TCP particulate graft material soaked in BMSCs and covered with a resorbable $\mathrm{CM}$ )

BV-NFB was significantly high at 2 weeks $\left((1.69 \pm 0.90) \mathrm{mm}^{3}\right)(P<$ $0.05), 4$ weeks $\left((4.32 \pm 0.13) \mathrm{mm}^{3}\right)(P<0.05), 6$ weeks $\left((7.14 \pm 1.10) \mathrm{mm}^{3}\right)$ $(P<0.01)$, and 10 weeks $\left((10.99 \pm 1.20) \mathrm{mm}^{3}\right)(P<0.001)$ compared to baseline $\left(0 \mathrm{~mm}^{3}\right)$. Compared to 2 weeks $(P<0.001)$, 4 weeks $(P<0.01)$, and 6 weeks $(P<0.01)$, BV-NFB was significantly high at 10 weeks (Table 3).

BMD-NFB was significantly high at 4 weeks $\left((0.18 \pm 0.01) \mathrm{g} \cdot \mathrm{mm}^{-3}\right)$ $(P<0.001), 6$ weeks $\left((0.31 \pm 0.04) \mathrm{g} \cdot \mathrm{mm}^{-3}\right)(P<0.01)$, and 10 weeks $\left((0.46 \pm 0.05) \mathrm{g} \cdot \mathrm{mm}^{-3}\right)(P<0.01)$ compared to baseline $\left(0 \mathrm{~g} \cdot \mathrm{mm}^{-3}\right)$. Compared to 2 weeks $\left((0.11 \pm 0.01) \mathrm{g} \cdot \mathrm{mm}^{-3}\right)$, BMD-NFB was significantly high at 4 weeks $(P<0.01), 6$ weeks $(P<0.01)$, and 10 weeks $(P<0.01)$ (Table 3).

There was no statistically significant difference in BV of remaining TCP particles at all time intervals. At 10 weeks, BMD-remaining TCP particles were significantly higher than that at 6 weeks $\left((0.17 \pm 0.02) \mathrm{g} \cdot \mathrm{mm}^{-3}\right)$ and 10 weeks $\left((0.23 \pm 0.04) \mathrm{g} \cdot \mathrm{mm}^{-3}\right)(P<0.01)$ compared to baseline $\left(0 \mathrm{~g} \cdot \mathrm{mm}^{-3}\right)$ (Table 3 and Figure $2 \mathrm{c}$ ).

\section{Rate of new bone formation within each group}

In group 1, the rate of new bone formation from baseline to 2 weeks, 2 weeks to 4 weeks, 4 weeks to 6 weeks, and 6 weeks to 10 weeks was 0.02 , $0.04,0.05$, and $0.06 \mathrm{~mm}^{3} \cdot \mathrm{d}^{-1}$, respectively (Table 4 ).

In group 2, new bone formed at a rate of $0.08,0.06,0.14$, and $0.11 \mathrm{~mm}^{3} \cdot \mathrm{d}^{-1}$ from baseline to 2 weeks, 2 weeks to 4 weeks, 4 weeks to 6 weeks, and 6 weeks to 10 weeks, respectively (Table 4).

In group 3, the rate of new bone formation from baseline to 2 weeks, 2 weeks to 4 weeks, 4 weeks to 6 weeks, and 6 weeks to 10 weeks was $0.12,0.18,0.20$, and $0.14 \mathrm{~mm}^{3} \cdot \mathrm{d}^{-1}$, respectively (Table 4 ).

\section{Histologic results}

- Group 1 (control group)

Edges of the CSD bridged by fibrous CT were seen with remnants of $\mathrm{CM}$ as a homogeneous acellular acidophilic material (Figure $3 \mathrm{a}$ ). NFB at the edge of the CSD with fibrous CT at the advancing edge of NFB was observed with a clear boundary between NFB and NB (Figure $3 b$ ). Osteocytes and blood vessels (BV) were evident in the matrix of NFB with fibrous CT and inflammatory cells on the advancing edge of NFB (Figure 3c).

- Group 2 (BMSCs filled in the critical size defect and covered with a resorbable $\mathrm{CM}$ )

Edges of the CSD bridged by a thin strip of NFB extending from one edge of the CSD were seen with an island of NFB positioned cranially along with CT (Figure 4a). NFB was evident on the edge and in the middle of the CSD with no evidence of inflammation (Figure $4 \mathrm{~b}$ ). Osteocytes and BV were also evident in the matrix of NFB with no evidence of inflammation (Figure 4c).

- Group 3 (TCP particulate graft soaked in BMSCs and covered with a resorbable CM)

Edges of the CSD were seen with a bridge of NFB extending into the center of the CSD from one edge of the CSD with a granular crystalline material (TCP) interspersed with fibrous CT (Figure 5a). Remnant TCP particles appeared as granular crystalline material interspersed with fibrous CT and encapsulated by inflammatory cells (Figure 5b). Osteocytes and BV were visible in the matrix of NFB (Figure $5 \mathrm{c}$ ).

\section{Hardness of NFB compared to native calvaria bone among groups 1, 2 , and 3}

In groups 1 and 2, hardness was similar to the natural bone (adjacent to the defect) with no significant difference between their mean values, 444.75 and $454.93 \mathrm{Mpa}$, respectively. In group 3, hardness was significantly less $(58.65 \mathrm{Mpa})$ compared to that in groups 1 and 2 (444.75 and $454.93 \mathrm{Mpa})$, respectively.

The mean reduced modulus of elasticity was similar between groups 1 and 2 (13.85 and $8.88 \mathrm{GPa}$, respectively). In group $3(109.11 \mathrm{GPa})$, this value was significantly higher than groups 1 and 2 (13.85 and $8.88 \mathrm{GPa}$, respectively) (Figures 6 and 7).

Table 3 Mean \pm standard deviation of bone volume and bone mineral density of newly formed bone and remaining bone particles in group 3

\begin{tabular}{|c|c|c|c|c|}
\hline Timeline & \multicolumn{2}{|c|}{ Newly formed bone } & \multicolumn{2}{|c|}{ Remaining bone particles } \\
\hline Baseline & $0 *{ }^{*}+w_{5}$ & $0^{1+0+4 s s}$ & $6.50 \pm 1.50$ & $0^{\text {ititat }}$ \\
\hline 4 weeks & {$\left[4.32 \pm 0.13^{\dagger \|}\right]_{* *}$} & $0.18 \pm 0.01^{1+\| * * *}$ & $4.90 \pm 1.00$ & $0.14 \pm 0.01$ \\
\hline 6 weeks & $* * 7.14 \pm 1.10^{*}$ & $0.31 \pm 0.04$ & $4.90 \pm 1.00$ & $0.17 \pm 0.02^{\dagger \dagger \dagger}$ \\
\hline 10 weeks & 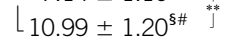 & $0.46 \pm 0.05^{\# \# \S \varsigma_{* * *}}$ & $3.46 \pm 0.80$ & $0.23 \pm 0.04$ 林ฐsฐ \\
\hline
\end{tabular}

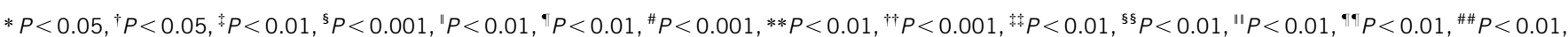
*** $P<0.01,{ }^{\dagger \dagger} P<0.01,{ }^{\text {t⿰木木 }} P<0.01,{ }^{\$ \S \S} P<0.05$. 

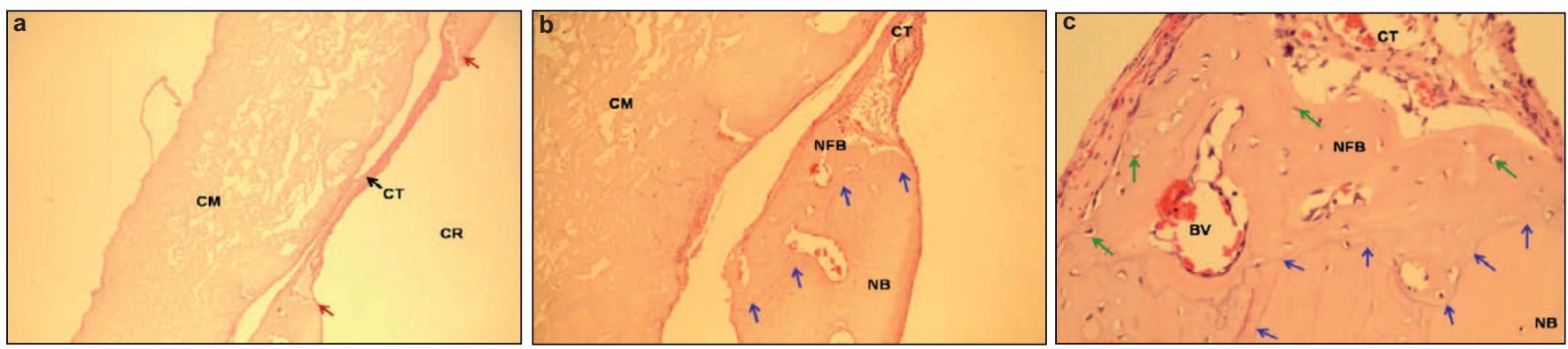

Figure 3 Histologic assessment (using hematoxylin-eosin staining) of CSD and native calvaria in group 1. (a) Edges of the CSD (red arrows) bridged by fibrous CT with remnants of $\mathrm{CM}(\times 4)$. (b) NFB at the edge of the CSD with fibrous CT at the advancing edge of NFB. Blue arrows show the boundary between NFB and NB with remnants of $\mathrm{CM}(\times 10)$. (c) Osteocytes (green arrows) and BVs are evident in the matrix of NFB with fibrous CT and inflammatory cells on the advancing edge of NFB. Blue arrows show the boundary between NFB and NB $(\times 40)$. BV, bone volume; CM, collagen membrane; CR, CSD, calvaria critical size defect; CT, connective tissue; $\mathrm{NB}$, native bone; NFB, newly formed bone.
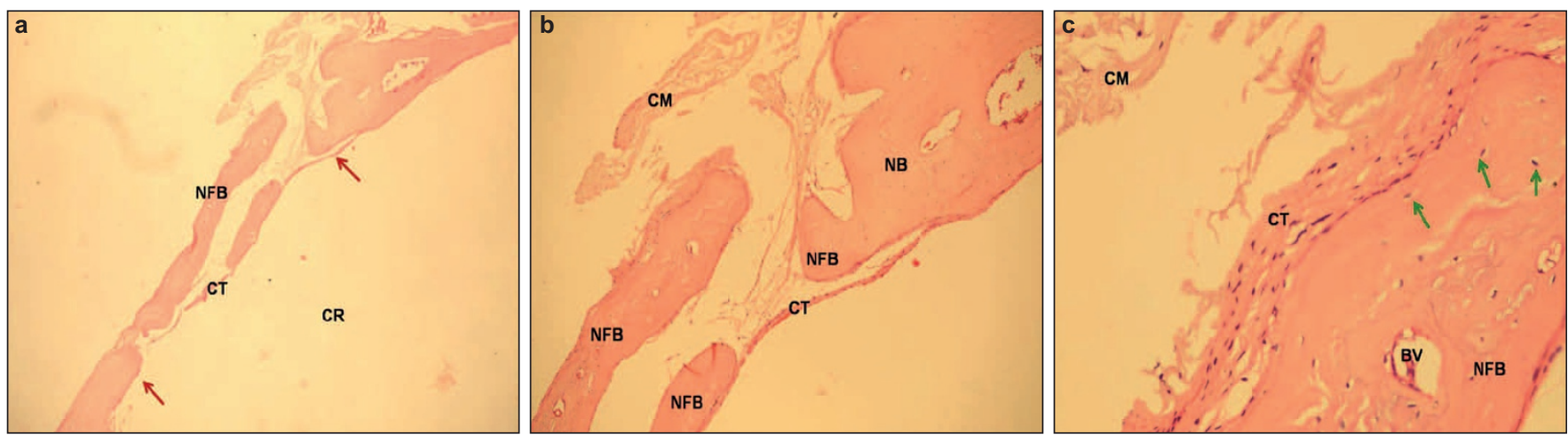

Figure 4 Histologic assessment (using hematoxylin-eosin staining) of CSD and native calvaria in group 2. (a) Edges of the CSD (red arrows) bridged by a thin strip of NFB extending from one edge of the CSD $(\times 4)$. (b) NFB seen on the edge and in the middle of the CSD with CT fibers bridging the NFB with the edge of the CSD. Remnants of $\mathrm{CM}$ can be seen as an acellular acidophilic material with no evidence of inflammation $(\times 10)$. (c) Osteocytes (green arrows) were evident in the matrix of NFB with fibrous CT adjacent to the leading edge of the NFB. There is no evidence of inflammation and remnants of CM were visible as an acellular acidophilic material $(\times 40)$. BV, bone volume; CM, collagen membrane; CR, CSD, calvaria critical size defect; CT, connective tissue; NB, native bone; NFB, newly formed bone.
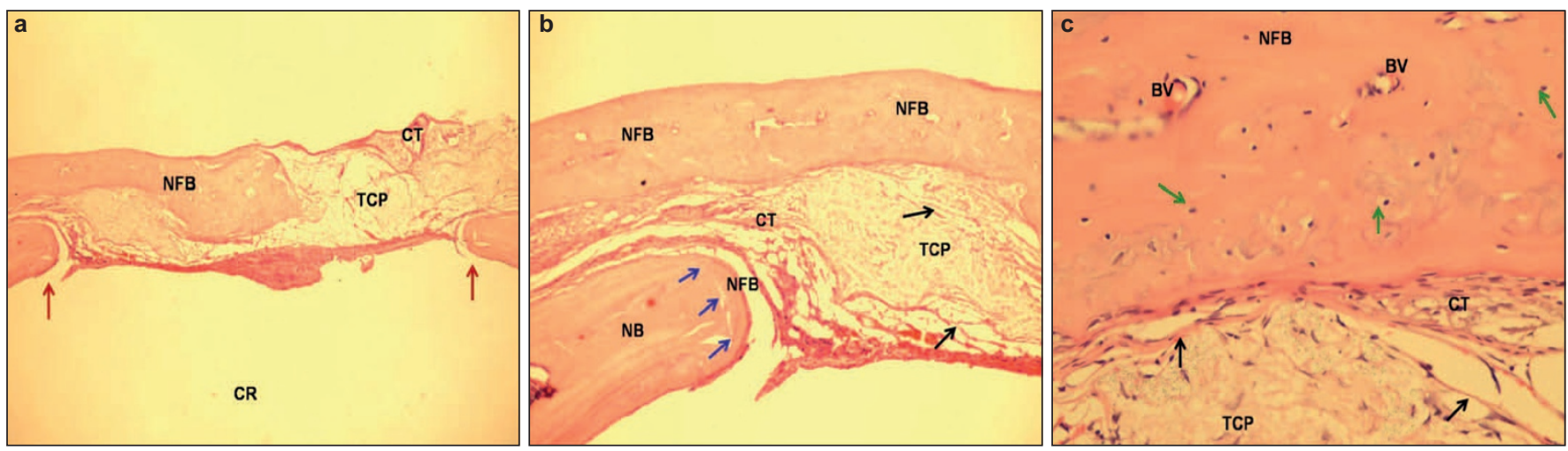

Figure 5 Histologic assessment (using hematoxylin-eosin staining) of CSD and native calvaria in group 3. (a) Edges of the CSD (red arrows) bridging the NFB with a granular crystalline material interspersed with fibrous CT $(\times 4)$. (b) Blue arrows show the boundary between the NFB and NB with remnants of particulate graft interspersed with fibrous CT and encapsulated by inflammatory cells (black arrows) $(\times 10)$. (c) Osteocytes (green arrows) were visible in the matrix of NFB remaining bone graft particles seen as a granular crystalline material along with inflammatory encapsulation (black arrows) $(\times 40)$. BV, bone volume; CM, collagen membrane; CR, CSD, calvaria critical size defect; CT, connective tissue; NB, native bone; NFB, newly formed bone; TCP, tricalcium phosphate. 

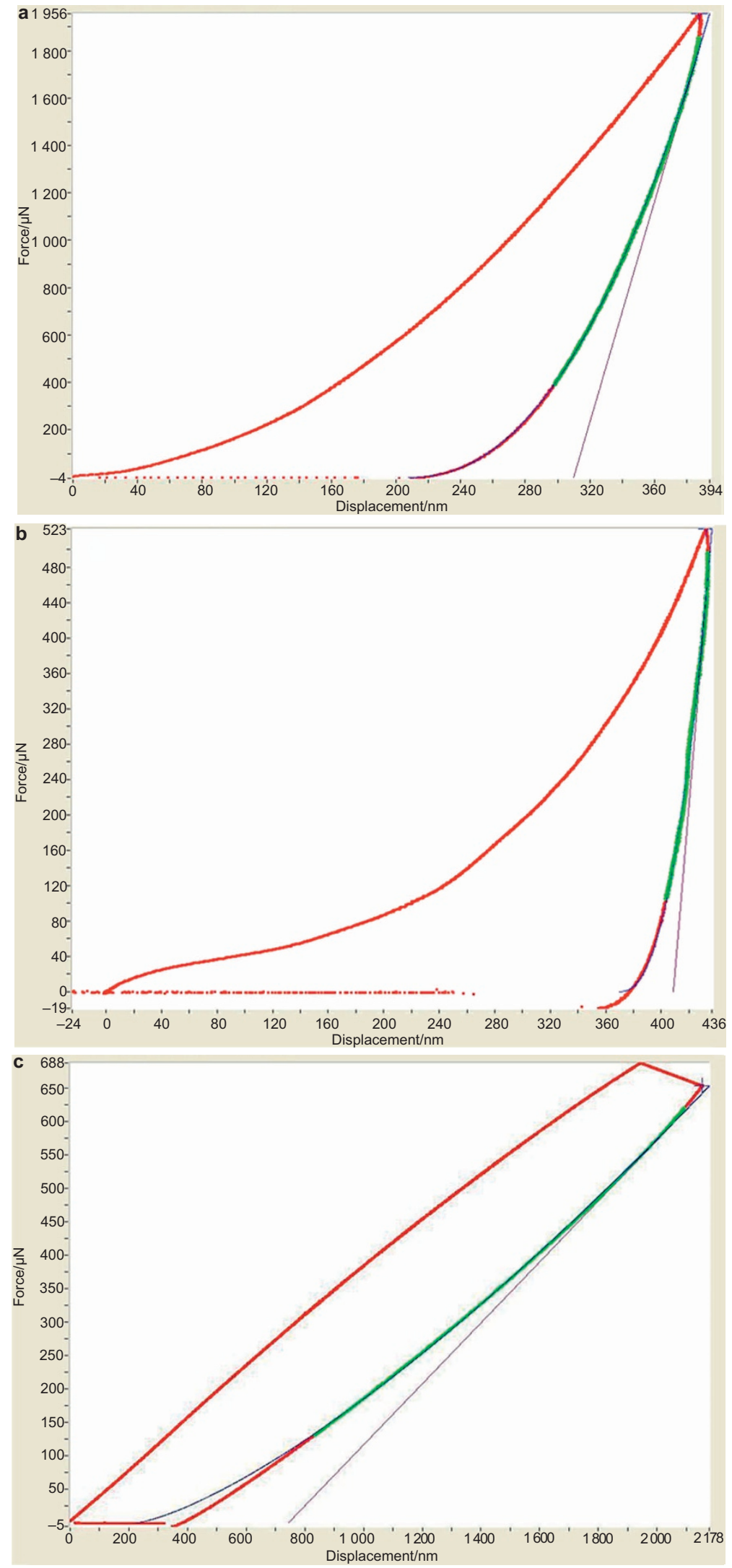

Figure 6 Load displacement curves and calculated hardness values $(\mathrm{H})$ generated from nanoindentation of calvarial bone of a rat. (a) Natural bone adjacent to the defect site; (b) regenerated bone with BMSC, TCP, and membrane; (c) regenerated bone with BMSC and membrane. BMSC, bone marrow-derived mesenchymal stem cell; TCP, tricalcium phosphate. 

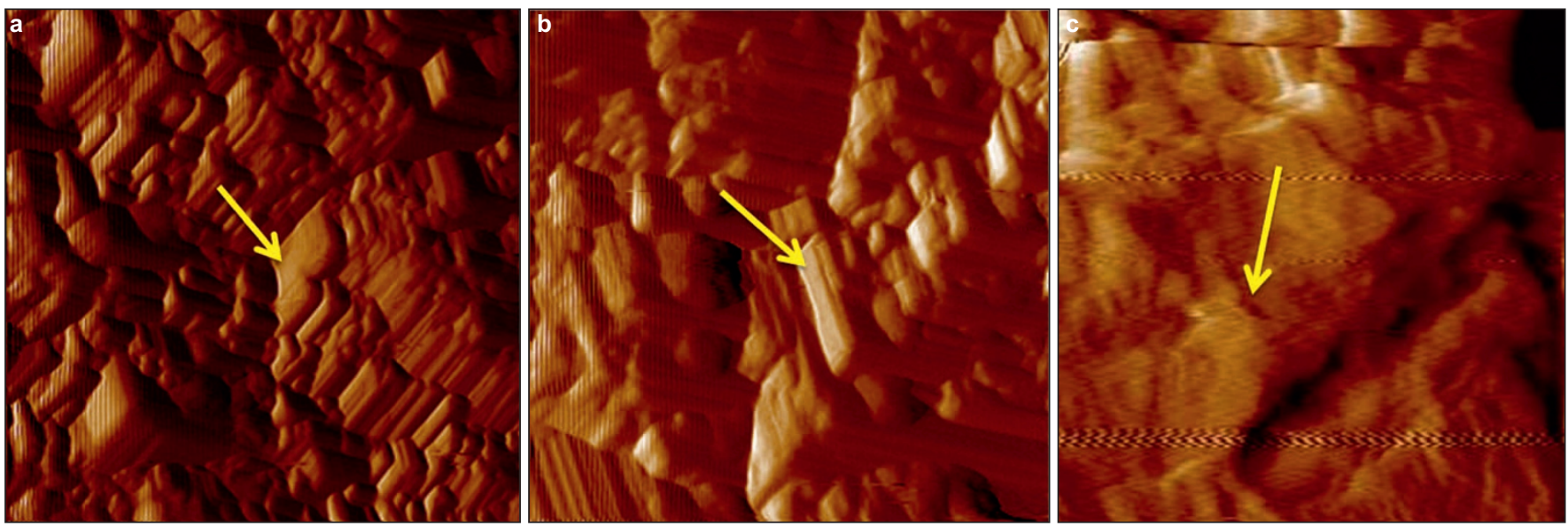

Figure 7 In situ SPM image of residual indent showing residual impression/or fracture events. This image was taken using the same indentation tip that performed the testing. (a) Natural adjacent bone to the calvarial defect; (b) regenerated bone following the use of BMSC, TCP, and membrane; (c) regenerated bone with BMSCs and membrane. BMSC, bone marrow derived mesenchymal stem cell; SPM, scanning probe microscopy; TCP, tricalcium phosphate.

Table 4 Rate of new bone formation among standardized critical size defects in groups 1,2 and 3

\begin{tabular}{lccc}
\hline & \multicolumn{3}{c}{ Rate of new bone formation $/\left(\mathrm{mm}^{3} \cdot \mathrm{d}^{-1}\right)$} \\
\cline { 2 - 4 } Timeline & Group 1 & Group 2 & Group 3 \\
\hline Baseline to 2 weeks & 0.02 & 0.08 & 0.12 \\
2 weeks to 4 weeks & 0.04 & 0.06 & 0.18 \\
4 weeks to 6 weeks & 0.05 & 0.14 & 0.20 \\
6 weeks to 10 weeks & 0.06 & 0.11 & 0.14 \\
\hline
\end{tabular}

\section{DISCUSSION}

The calvarium is considered as a suitable location for assessing bone augmentation because of its embryological and morphological similarities with bones in the oral and maxillofacial region. ${ }^{24}$ In addition, it is also acknowledged that osteoprogenitor cells and vascular supply are among the factors that influence extra-cortical bone regeneration. ${ }^{25}$ Previous ex vivo studies have applauded the contribution of BMSCs in enhancing bone formation in GBR protocols; ${ }^{14,26}$ however, it is pertinent to mention that in the present in vivo $\mu \mathrm{CT}$, we evaluated the potential of BMSCs to regenerate bone in an established rat calvarium model in real time. In the present real-time in vivo $3 \mathrm{D} \mu \mathrm{CT}$ study, the evidence of NFB in CSD treated with BMSCs (with or without TCP graft) appeared as early as 2 weeks following GBR. The rate of new bone formation among CSD in groups 2 and 3 were approximately 2.8 and 4 times, respectively higher than the rate of new bone formation in the control group (group 1). It is however notable that the rate of new bone formation in groups 2 and 3 at $4-6$ weeks $\left(0.14\right.$ and $0.2 \mathrm{~mm}^{3} \cdot \mathrm{d}^{-1}$, respectively) were rather similar. The proposed mechanisms via which BMSCs contribute to enhancement in bone regeneration encompass a direct pathway by maturation into osteoblasts in vivo or an indirect pathway by paracrine effects on host stem or progenitor cells. ${ }^{27}$ Since our real time in vivo $\mu \mathrm{CT}$ results showed evidence of NFB by the second week of GBR, this implies that transformation of BMSCs into osteoblasts occurs by this time after introduction into CSDs. It has been shown in vitro that initial osteogenic differentiation in BMSCs takes approximately 14 days. ${ }^{28-30}$ Moreover, in vitro results have also shown that by the 14th day of cellular differentiation, calcium and phosphate deposition initiates thereby forming new mineralized tissues. ${ }^{28-30}$ The present real-time in vivo $\mu \mathrm{CT}$ findings support these results.

It has been reported that a communication network exists between osteocytes and osteoblasts which produces an osteogenic response in BMSCs. ${ }^{14}$ In addition, there is sufficient evidence to prove that osteocytes act as the mechano-transducers for bone and also actively participate in regulating osteoclast formation from monocyte precursors. ${ }^{31-32}$ However, Birmingham et al. ${ }^{28}$ showed that osteocytes are more influential in producing biochemical signals that stimulate osteogenesis in BMSCs than osteoblasts. ${ }^{28}$ This is probably because osteocytes express high quantities of osteocalcin as compared to osteoblasts and it occurs between 2 and 4 weeks of BMSC culturing in vitro. ${ }^{33}$ Our 10-week histologic results showed osteocytes entrapped in the bone matrix among groups treated with BMSCs compared to the control group where no histologic evidence of the presence of osteocytes was observed. It is likely that on our study, the BMSCs started differentiating into osteocytes by the 2nd week of surgery and probably by the 4 th week of BMSC implantation and there were significant numbers of osteocytes in the developing bone matrix. Since our study design was based on in vivo calvarial $\mu \mathrm{CT}$ assessment up to the 10th postoperative week, we were unable to perform histologic assessments of calvaria at earlier stage of bone formation to identify osteocytes in the developing bone matrix; hence, further studies are warranted in this regard.

It is pertinent to mention that alloplastic particulate bone graft materials are unable to produce bony trabeculae (as in the case of natural bone), and are amorphous. Moreover, TCP starts undergoing resorption as early as 6 months after placement in osseous defects and also forms a weak connection between bone and implant. ${ }^{34-35}$ The present results showed that the hardness of NFB was similar to that of the NB in groups 1 and 2 as compared to the NFB in group 3. Likewise, values for the modulus of elasticity were also significantly high in group 3 compared to groups 1 and 2. This suggests that TCP, when used in combination with BMSCs, and with CM, was unable to form bone of significant strength that could possibly provide mechanical "lock" between the natural bone and NFB.

Within the limits of the present experiment, it is concluded that use of BMSCs as adjuncts to conventional GBR initiated new bone formation as early as 2 weeks of treatment compared to when GBR is attempted without adjunct BMSC therapy.

\section{ACKNOWLEDGEMENTS}

The project was full financially supported by King Saud University, through Vice Deanship of Research Chairs. 
1 Mellonig JT, Valderrama Mdel P, Cochran DL. Histological and clinical evaluation of recombinant human platelet-derived growth factor combined with beta tricalcium phosphate for the treatment of human class III furcation defects. Int J Periodontics Restorative Dent 2009; 29(2): 169-177.

2 Urban IA, Jovanovic SA, Lozada JL. Vertical ridge augmentation using guided bone regeneration (GBR) in three clinical scenarios prior to implant placement: retrospective study of 35 patients 12 to 72 months after loading. Int J Oral Maxillofac Implants 2009; 24(3): 502-510.

3 Proussaefs $P$, Lozada J. The use of resorbable collagen membrane in conjunction with autogenous bone graft and inorganic bovine mineral for buccal/labial alveolar ridge augmentation: a pilot study. J Prosthet Dent 2003; 90(6): 530-538.

4 Hämmerle $\mathrm{CH}$, Jung RE, Yaman D et al. Ridge augmentation by applying bioresorbable membranes and deproteinized bovine bone mineral: a report of twelve consecutive cases. Clin Oral Implants Res 2008; 19(1): 19-25.

5 Hämmerle $\mathrm{CH}$, Lang NP. Single stage surgery combining transmucosal implant placement with guided bone regeneration and bioresorbable materials. Clin Oral Implants Res 2001; 12(1): 9-18.

6 Nevins M, Nevins ML, Schupbach $\mathrm{P}$ et al. A prospective, randomized controlled preclinical trial to evaluate different formulations of biphasic calcium phosphate in combination with a hydroxyapatite collagen membrane to reconstruct deficient alveolar ridges. J Oral Implantol 2013; 39(2): 133-139.

7 Saffar JL, Colombier ML, Detienville R. Bone formation in tricalcium phosphate-filled periodontal intrabony lesions. Histological observations in humans. J Periodontol 1990; 61(4): 209-216.

8 Stahl SS, Froum S. Histological evaluation of human intraosseous healing responses to the placement of tricalcium phosphate ceramic implants. I. Three to eight months. J Periodontol 1986; 57(4): 211-217.

9 Bowers GM, Vargo JW, Levy B et al. Histologic observations following the placement of tricalcium phosphate implants in human intrabony defects. J Periodontol 1986, 57(5): 286-287.

10 Schenk RK, Buser D, Hardwick WR et al. Healing pattern of bone regeneration in membrane-protected defects: a histologic study in the canine mandible. Int J Oral Maxillofac Implants 1994; 9(1): 13-29.

11 Friedenstein AJ, Chailakhjan RK, Lalykina KS. The development of fibroblast colonies in monolayer cultures of guinea-pig bone marrow and spleen cells. Cell Tissue Kinet 1970; 3(4): 393-403.

12 Gimbel M, Ashley RK, Sisodia M et al. Repair of alveolar cleft defects: reduced morbidity with bone marrow stem cells in a resorbable matrix. J Craniofac Surg 2007; 18(4): 895-901.

13 Zigdon-Giladi H, Bick T, Lewinson D et al. Co-transplantation of endothelial progenitor cells and mesenchymal stem cells promote neovascularization and bone regeneration. Clin Implant Dent Relat Res 2015; 17(2): 353-359.

14 Zigdon-Giladi H, Lewinson D, Bick T et al. Mesenchymal stem cells combined with barrier domes enhance vertical bone formation. J Clin Periodontol 2013; 40(2) 196-202.

15 Camelo M, Nevins ML, Schenk RK et al. Clinical, radiographic, and histologic evaluation of human periodontal defects treated with Bio-Oss and Bio-Gide. Int J Periodontics Restorative Dent 1998; 18(4): 321-331.

16 Nevins ML, Camelo M, Lynch SE et al. Evaluation of periodontal regeneration following grafting intrabony defects with bio-oss collagen: a human histologic report. Int J Periodontics Restorative Dent 2003; 23(1): 9-17.

17 Nevins M, Nevins ML, Karimbux $N$ et al. The combination of purified recombinant human platelet-derived growth factor-BB and equine particulate bone graft for periodontal regeneration. J Periodontol 2012; 83(5): 565-573.

18 Al-Hezaimi K, lezzi G, Rudek I et al. Histomorphometric analysis of bone regeneration using a dual layer of membranes (dPTFE placed over collagen) in fresh extraction sites: a canine model. J Oral Implantol 2015; 41(2): 188-195.
19 Al-Hazmi BA, Al-Hamdan KS, Al-Rasheed A et al. Efficacy of using PDGF and xenograft with or without collagen membrane for bone regeneration around immediate implants with induced dehiscence-type defects: a microcomputed tomographic study in dogs. J Periodontol 2013; 84(3): 371-378.

20 Al-Hezaimi K, Al-Shabeeb MS, Al-Askar M et al. Microcomputed tomographic analysis of the alveolar ridge alteration around extraction sites with and without immediate implants placement: in vivo study. Clin Implant Dent Relat Res 2014; 16(2): 223-229.

21 Al-Askar M, O'Neill R, Stark PC et al. Effect of single and contiguous teeth extractions on alveolar bone remodeling: a study in dogs. Clin Implant Dent Relat Res 2013; 15(4): 569-575.

22 Fischer AH, Jacobson KA, Rose J et al. Hematoxylin and eosin staining of tissue and cell sections. CSH Protoc 2008; 2008: pdb. prot4986.

23 Schmid J, Wallkamm B, Hämmerle $\mathrm{CH}$ et al. The significance of angiogenesis in guided bone regeneration. A case report of a rabbit experiment. Clin Oral Implants Res 1997; 8(3): 244-248.

24 Dodde R 2nd, Yavuzer R, Bier UC et al. Spontaneous bone healing in the rabbit. J Craniofac Surg 2000; 11(4): 346-349.

25 Kanczler JM, Oreffo RO. Osteogenesis and angiogenesis: the potential for engineering bone. Eur Cell Mater 2008; 15: 100-114.

26 Zigdon-Giladi H, Bick T, Lewinson D et al. Mesenchymal stem cells and endothelial progenitor cells stimulate bone regeneration and mineral density. J Periodonto/2014; 85(7): 984-990.

27 Zachos T, Diggs A, Weisbrode S et al. Mesenchymal stem cell-mediated gene delivery of bone morphogenetic protein-2 in an articular fracture model. Mol Ther 2007; 15(8): 1543-1550.

28 Birmingham E, Niebur GL, McHugh PE et al. Osteogenic differentiation of mesenchymal stem cells is regulated by osteocyte and osteoblast cells in a simplified bone niche. Eur Cell Mater 2012; 23: 13-27.

29 Hoemann CD, El-Gabalawy H, McKee MD. In vitro osteogenesis assays: influence of the primary cell source on alkaline phosphatase activity and mineralization. Pathol Biol 2009; 57(4): 318-323.

30 Aubin JE. Regulation of osteoblast formation and function. Rev Endocr Metab Disord 2001; 2(1): 81-94.

31 Burra S, Nicolella DP, Francis WL et al. Dendritic processes of osteocytes are mechanotransducers that induce the opening of hemichannels. Proc Natl Acad Sci U S A 2010; 107(31): 13648-13653.

32 You L, Temiyasathit S, Lee P et al. Osteocytes as mechanosensors in the inhibition of bone resorption due to mechanical loading. Bone 2008; 42(1): 172-179.

33 Birnbaum RS, Bowsher RR, Wiren KM. Changes in IGF-I and -II expression and secretion during the proliferation and differentiation of normal rat osteoblasts. J Endocrinol 1995; 144(2): 251-259.

34 Srivastava AK, Libanati C, Oberrauch W et al. Analytical and clinical evaluation of the Bio-Rad HPLC kit for measurement of type I collagen cross links. J Bone Miner Metab 2003; 21(3): 134-144.

35 Khoshzaban A, Mehrzad S, Tavakoli V et al. The comparative effectiveness of demineralized bone matrix, beta-tricalcium phosphate, and bovine-derived anorganic bone matrix on inflammation and bone formation using a paired calvarial defect model in rats. Clin Cosmet Investig Dent 2011; 3: 69-78.

\footnotetext{
(c) (i) (8) $\Theta$ This work is licensed under a Creative Commons Attribution-

NonCommercial-NoDerivs 4.0 Unported License. The images or other third party material in this article are included in the article's Creative Commons license, unless indicated otherwise in the credit line; if the material is not included under the Creative Commons license, users will need to obtain permission from the license holder to reproduce the material. To view a copy of this license, visit http://creativecommons.org/licenses/ by-nc-nd/4.0/
} 